\title{
A Numerical Approximation Approach for Quantum Optimal Control of Two-Level Systems
}

\author{
Cutberto Romero-Meléndez ${ }^{1 *}$, Leopoldo González-Santos ${ }^{2}$ \\ 1 Department of Basic Sciences. Autonomous Metropolitan University, Avenida San Pablo 180, colonia \\ Reynosa T. Ciudad de México. 02200. México. \\ 2 Institute of Neurobiology. National Autonomous University of México, México. \\ * Corresponding author. Tel.: (52)55 51190909; email: cutberto@correo.azc.uam.mx \\ Manuscript submitted May 5, 2017; accepted June 28, 2017. \\ doi: 10.17706/ijapm.2017.7.4.268-274
}

\begin{abstract}
In this paper, we discuss a quantum control algorithm, devised by the authors, inspired in Rabitz and Krotov algorithms and applied to Nuclear Magnetic Resonance (NMR) That algorithm is based on a numerical method for iterative optimization. Specifically, we address the determination of external optimal pulses (controls) to minimal cost, over a two-level quantum system. We use the numerical approximation to find the optimal controls in the case of two external electromagnetic fields, integrating the associated equations of the Pontryagin Maximum Principle. That algorithm unifies and generalizes the Rabitz and Krotov algorithms. We compare the efficiency of these algorithms with the solutions found by analytical methods.
\end{abstract}

Key words: Lie algebra, nuclear magnetic resonance, optimal control, quantum control systems, iterative optimization.

\section{Introduction}

In recent years Optimal Geometric Control techniques have been used in Nuclear Magnetic Resonance (NMR) for the control of dynamics of quantum systems [1]. A fundamental problem in NMR is the determination of the minimum time required to perform a unitary transformation in a quantum system.

A sample is placed in an uniform and longitudinal static magnetic field $\mathcal{B}_{Z}$ in the direction of the axis $\mathrm{Z}$, aligning the magnetic moments of this sample. Then it is exposed to variable radio frequency fields in the $\mathrm{X}-\mathrm{Y}$ plane, $u_{x}(t), u_{y}(t)$, absorbing the energy through the sequence of transverse magnetic pulses. The total magnetic field to which the sample is subjected is $\overrightarrow{\mathcal{B}}(t)=\left(u_{x}(t), u_{y}(t), \mathcal{B}_{z}\right)$.

When the magnetic moment vector of the system is transferred to the $\mathrm{XY}$ plane the sequence of transverse magnetic pulses is stopped, making precess the magnetic moment vector. Repetitions of this process cause fluctuations in $\mathcal{B}_{z}$ and eventually cause decoherence. The pulse sequence should be as short as possible to minimize the effects of relaxation, to optimize the sensitivity to the experiment and to optimize the contrast of the image obtained. This is achieved by controlling the sequence of pulses that create a unitary transformation in the shortest possible time. For the Control Theory the minimization in time of a sequence of pulses equals the minimization of lengths of trajectories (in homogeneous spaces).

Consider a quantum system described by his wave function $\vec{\psi}(t)$ called state. The evolution of $\vec{\psi}(t)$ is determined by the Schrödinger equation 


$$
\frac{d}{d t} \vec{\psi}(t)=-i H(u(t)) \vec{\psi}(t)
$$

where $\vec{\psi}=\left(\psi_{1}, \psi_{2}\right):[0, T] \rightarrow \mathbb{C}^{2}$ is a vector representing the unitary ket $|\psi\rangle, T \in \mathbb{R}$ is the complete time of control, let's say $T=\frac{\pi}{\sqrt{2}}$, the Lebesgue integrable function $u:[0, T] \rightarrow \mathbb{C}^{2}$ is the intensity of the control field laser pulse and $H(t)$ is the Hamiltonian that represents the energy of the interaction of the spin angular momentum with the external magnetic field, therefore

$$
H(u(t))=-\gamma \mathcal{S}_{z} \mathcal{B}_{z}-\gamma \mathcal{S}_{x} u_{x}(t)-\gamma \mathcal{S}_{y} u_{y}(t)
$$

where $\overrightarrow{\mathcal{S}}=\left(\mathcal{S}_{x}, \mathcal{S}_{y}, \mathcal{S}_{z}\right)$ is the spin angular momentum operator and $\gamma$ is the gyromagnetic ratio of the system [2].

We study the simplest control system in a spin $-\frac{1}{2}$ particle interacting with the magnetic field. We consider the following optimal control problem for the pure state:

$$
\begin{aligned}
\frac{d}{d t} \vec{\psi}(t) & =-i H(u(t)) \vec{\psi}(t) \\
\vec{\psi}(0) & =\left(\begin{array}{l}
1 \\
0
\end{array}\right)
\end{aligned}
$$

The target state is $\vec{\psi}\left(\frac{\pi}{\sqrt{2}}\right)=\left(\begin{array}{l}0 \\ i\end{array}\right)$. This means that the spin turns $2 \pi$ radians from his initial position. Using an adjoint state (Lagrange multiplier) $\lambda(t)$ we obtain

$$
\begin{gathered}
\frac{d}{d t} \lambda(t)=\left(\tilde{S}_{z}+u_{y}(t) \tilde{S}_{y}+u_{x}(t) \tilde{S}_{x}\right) \lambda(\mathrm{t}) \\
\lambda\left(\frac{\pi}{\sqrt{2}}\right)=\left(\begin{array}{l}
0 \\
i
\end{array}\right)
\end{gathered}
$$

The development of monotonic algorithms applied to Quantum Control Theory has been generating approximative procedures to get the sequences of pulses in a state transfer problem in a single spin.

There exist several optimization procedures to compute the approximate solution of (3), by example, Rabitz-Zhu algorithm, Krotov et al. algorithm and Maday-Turinici algorithm, used on Quantum Molecular Dynamics. However, this type of algorithms can be used in the Optimal Control framework. A way to control the system is to light it with a laser pulse, so $u(t)$ is the intensity of the control laser pulse. That procedures compute iteratively sequences $\left\{\vec{\psi}^{(k)}(t), u_{x}^{(k)}(t), u_{y}^{(k)}(t), \lambda^{(k)}(t)\right\}, k \in \mathbb{N} \quad$ and, solving repeatedly the Schrödinger equation, approximate the solution $\left\{\vec{\psi}(t), u_{x}(t), u_{y}(t), \lambda(t)\right\}$.

It's important to use an algorithm with an appropriate performance to solve the control quantum equations (3), minimizing the cost $J\left(u_{x}, u_{y}\right)=\left\langle\vec{x}^{t}\left(\frac{\pi}{\sqrt{2}}\right)|O| \vec{x}\left(\frac{\pi}{\sqrt{2}}\right)\right\rangle+\int_{0}^{\frac{\pi}{\sqrt{2}}}\left(u_{x}^{2}(t)+u_{y}^{2}(t)\right) d t$, where $O$ is the matrix observable with target information, which will allow us an optimum evolution of the system.

\section{Problem Formulation}

\subsection{Settings}

We have adapted two iterative algorithms due to Krotov et al. and Rabitz et al. respectively, to optimize a 
system with two controls (pulses of external magnetic fields) restricted to minimum cost. We present that algorithm and we done a demonstration of convergence.

We consider the following optimal control problem, where we have changed the complex problem (3) into a real problem [3]:

$$
\begin{gathered}
\frac{d}{d t} \vec{x}(t)=\left(\tilde{S}_{z}+u_{y}(t) \tilde{S}_{y}+u_{x}(t) \tilde{S}_{x}\right) \vec{x}(\mathrm{t}) \\
\vec{x}(0)=\left(\begin{array}{l}
1 \\
0 \\
0 \\
0
\end{array}\right)
\end{gathered}
$$

where $\tilde{S}_{x}, \tilde{S}_{y}$ and $\tilde{S}_{z}$ are the realification of Pauli matrices: $S_{x}, S_{y}, S_{z}$ :

$$
\tilde{S}_{x}=\left(\begin{array}{cccc}
0 & 0 & 0 & 1 \\
0 & 0 & 1 & 0 \\
0 & -1 & 0 & 0 \\
-1 & 0 & 0 & 0
\end{array}\right) \quad \tilde{S}_{y}=\left(\begin{array}{cccc}
0 & -1 & 0 & 0 \\
1 & 0 & 0 & 0 \\
0 & 0 & 0 & -1 \\
0 & 0 & 1 & 0
\end{array}\right) \quad \tilde{S}_{z}=\left(\begin{array}{cccc}
0 & 0 & 0 & 1 \\
0 & 0 & 1 & 0 \\
0 & -1 & 0 & 0 \\
-1 & 0 & 0 & 0
\end{array}\right)
$$

And

$$
\psi=\left(\psi_{1}, \psi_{2},\right)^{T}:[0, \mathrm{~T}] \rightarrow \mathbb{C}^{2}, \vec{x}(\mathrm{t}):=\left(\begin{array}{l}
\operatorname{Re}\left(\Psi_{1}\right) \\
\operatorname{Re}\left(\psi_{2}\right) \\
\operatorname{Im}\left(\psi_{1}\right) \\
\operatorname{Im}\left(\psi_{2}\right)
\end{array}\right)
$$

Given the final state

$$
\vec{x}\left(\frac{\pi}{\sqrt{2}}\right)=\left(\begin{array}{l}
0 \\
0 \\
0 \\
1
\end{array}\right)
$$

minimizing the functional cost

$$
J\left(u_{x}, u_{y}\right)=\left\langle\vec{x}^{t}\left(\frac{\pi}{\sqrt{2}}\right)|O| \vec{x}\left(\frac{\pi}{\sqrt{2}}\right)\right\rangle+\int_{0}^{\frac{\pi}{\sqrt{2}}}\left(u_{x}^{2}(t)+u_{y}^{2}(t)\right) d t
$$

where $O$ is the matrix:

$$
O=\vec{x}\left(\frac{\pi}{\sqrt{2}}\right) \vec{x}^{t}\left(\frac{\pi}{\sqrt{2}}\right)
$$

Since $\operatorname{span}\left\{\mathcal{S}_{x}, \mathcal{S}_{y}, \mathcal{S}_{z}\right\}=\mathfrak{s u}(2)$, where $\mathfrak{s u}(2)$ is the Lie algebra of traceless anti-Hermitian $2 \times 2$ matrices and $\mathcal{S}_{x}, \mathcal{S}_{y}, \mathcal{S}_{z}$ are orthogonal and linearity independent, the existence of the optimal control for the system (4) with the final condition (9) is assured [4].

The problem is the determination of the optimal controls to perform a unitary spin evolution of the state spin 1/2 to State spin $-1 / 2$, with minimum cost (9), in a two-level quantum system.

We have devised an algorithm based on the algorithms of Rabitz et al., Krotov et al. and Maday-Turinici, 
which unifies and generalizes them for the case of two controls $u_{x}(t), u_{y}(t)$.

\subsection{Recursion Formulas}

Given $\delta_{1}, \delta_{2}, \eta_{1}$ and $\eta_{2} \in[0,2]$, functions $\lambda^{0}(t), v^{0}(t), w^{0}(t)$ and $k \in \mathbb{N}, k \geq 1$

$$
\begin{aligned}
& \frac{d}{d t} \vec{x}^{(k)}(t)=\left(\begin{array}{cccc}
0 & -u_{y}^{(k)}(t) & -1 & -u_{x}^{(k)}(t) \\
u_{y}^{(k)}(t) & 0 & u_{x}^{(k)}(t) & 1 \\
1 & u_{x}^{(k)}(t) & 0 & -u_{y}^{(k)}(t) \\
u_{x}^{(k)}(t) & -1 & u_{y}^{(k)}(t) & 0
\end{array}\right) \vec{x}^{(k)}(t) \\
& \vec{x}^{(k)}(0)=\left(\begin{array}{l}
1 \\
0 \\
0 \\
0
\end{array}\right) \\
& u_{y}^{(k)}(t)=\left(1-\delta_{1}\right) v^{(k-1)}(t)+\delta_{1} \lambda^{T^{(k-1)}}(t) \tilde{S}_{x} \vec{x}^{(k)}(t) \\
& u_{x}^{(k)}(t)=\left(1-\delta_{2}\right) w^{(k-1)}(t)+\delta_{2} \lambda^{T^{(k-1)}}(t) \tilde{S}_{y} \vec{x}^{(k)}(t) \\
& \frac{d}{d t} \lambda^{(k)}(t)=\left(\begin{array}{cccc}
0 & -v^{(k)}(t) & -1 & -w^{(k)}(t) \\
v^{(k)}(t) & 0 & -w^{(k)}(t) & 1 \\
1 & w^{(k)}(t) & 0 & -v^{(k)}(t) \\
w^{(k)}(t) & -1 & v^{(k)}(t) & 0
\end{array}\right) \lambda^{(k)}(t) \\
& \lambda^{(k)}\left(\frac{\pi}{\sqrt{2}}\right)=\left(\begin{array}{c}
0 \\
0 \\
0 \\
x_{4}^{(k)}\left(\frac{\pi}{\sqrt{2}}\right)
\end{array}\right) \\
& v^{(k)}(t)=\left(1-\eta_{1}\right) u_{y}^{(k)}(t)+\eta_{1} \lambda^{T^{(k)}}(t) \tilde{S}_{y} \vec{x}^{(k)}(t) \\
& w^{(k)}(t)=\left(1-\eta_{2}\right) u_{x}^{(k)}(t)+\eta_{2} \lambda^{T^{(k)}}(t) \tilde{S}_{x} \vec{x}^{(k)}(t)
\end{aligned}
$$

\subsection{Algorithm}

The following algorithm allows to find the optimal controls $u_{x}, u_{y}$ of the problem (4), minimizing the cost $J\left(u_{x}, u_{y}\right)$ :

1) Choice $\lambda^{0}(t), v^{0}(t), w^{0}(t)$.

2) Choice the values $\delta_{1}, \delta_{2}, \eta_{1}$ and $\eta_{2} \in[0,2]$.

3) Replace $\delta_{1}, \lambda^{0}(t), v^{0}(t)$ in (12) to get $u_{y}^{(1)}(t)$.

4) Replace $\delta_{2}, \lambda^{0}(t), w^{0}(t)$ in (13) to get $u_{x}^{(1)}(t)$.

5) In (16) replace $u_{y}^{(1)}(t)$ and $\eta_{1}$.

6) Replace $u_{x}^{(1)}(t)$ and $\eta_{2}$ in (17).

7) Integrate (11) forward to get $\vec{x}^{(1)}(t)$ using $u_{x}^{(1)}(t)$ and $u_{y}^{(1)}(t)$. 
8) Integrate (14) backwards to get $\lambda^{(1)}(t)$ using $u_{x}^{(1)}(t)$ and $u_{y}^{(1)}(t)$.

9) Replace $\vec{x}^{(1)}(t)$ in the equation to $u_{y}^{(1)}(t)$.

10) $\left\{\lambda^{(k+1)}(t), v^{(k+1)}(t), w^{(k+1)}(t)\right\} \rightarrow\left\{\lambda^{(k)}(t), v^{(k)}(t), w^{(k)}(t)\right\}$

11) $\left\{u_{x}^{(k+1)}(t), u_{y}^{(k+1)}(t)\right\} \rightarrow\left\{u_{x}^{(k)}, u_{y}^{(k)}(t)\right\}$

12) Continue until convergence.

\section{Results}

The initial choice was $\delta_{1}=0.5, \delta_{2}=0.5, \eta_{1}=1.5, \eta_{2}=1.5, v^{0}(t)=\cos t, w^{0}(t)=1$. The process was convergent at $k=15$ and the cost was $J=0.8492$

The second choice was $\delta_{1}=0.5, \delta_{2}=1.5, \eta_{1}=1.5, \eta_{2}=0.5, v^{0}(t)=\cos t, w^{0}(t)=\cos t$. The process was convergent at $k=15$ and the cost was again $J=0.8492$

\section{Optimal Controls}

We present the optimal controls limit functions (Fig. 1) and the limit functional cost (Fig. 2) in the iterative process.
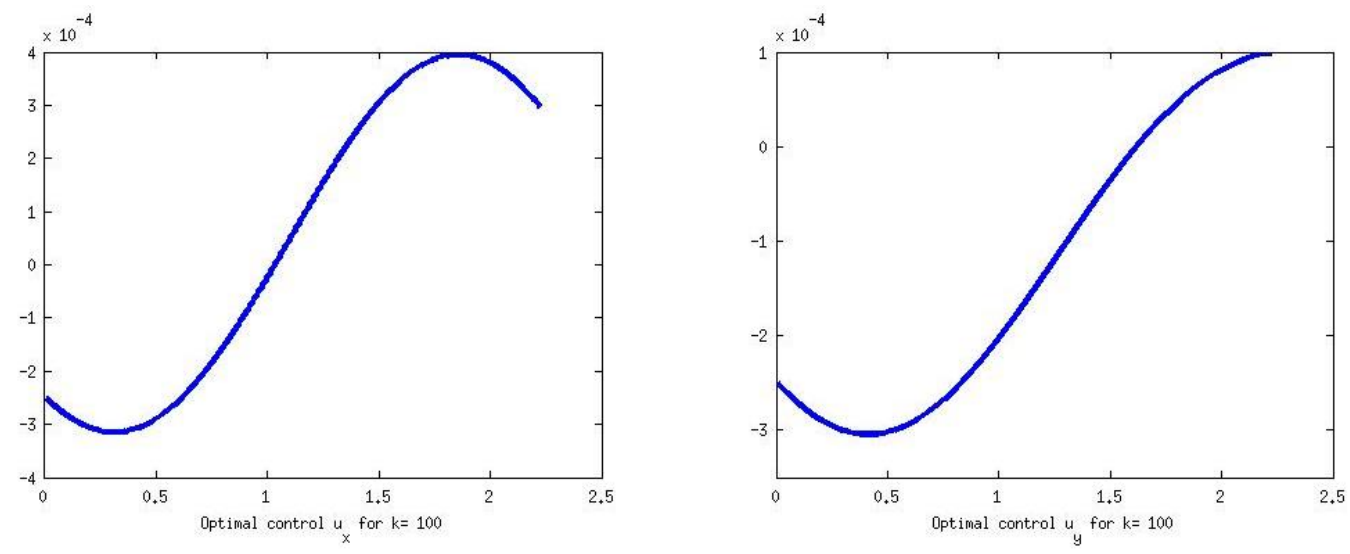

Fig. 1. The optimal controls $u_{x}(t), u_{y}(t)$ for $k=100$.

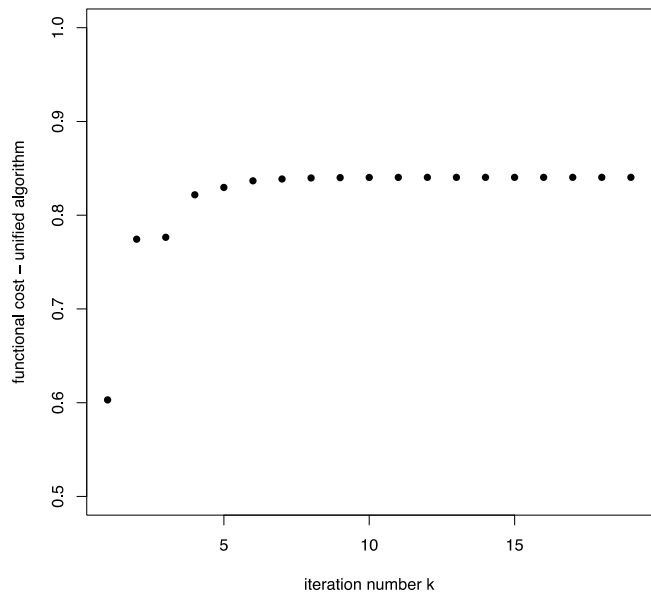

Fig. 2. The functional cost convergence.

\subsection{Convergence}

We have the following result about the monotonic convergence of our algorithm:

\section{Theorem}


The algorithm converges monotonically:

$$
J\left(u_{x}^{(k+1)}, u_{y}^{(k+1)}\right) \geq J\left(u_{x}^{(k)}, u_{y}^{(k)}\right), \quad \forall k \in \mathbb{N}
$$

\section{Proof.}

The proof is based on [3], for the case of two controls:

$$
\begin{aligned}
J\left(u_{x}^{(k+1)}, u_{y}^{(k+1)}\right)-J\left(u_{x}^{(k)}, u_{y}^{(k)}\right) & =\left\langle\left(\vec{x}^{(k+1)}\right)^{t}\left(\frac{\pi}{\sqrt{2}}\right)|O| \vec{x}^{(k+1)}\left(\frac{\pi}{\sqrt{2}}\right)\right\rangle+\int_{0}^{\frac{\pi}{\sqrt{2}}}\left(u_{x}^{(k+1)^{2}}(t)+u_{y}^{(k+1)^{2}}(t)\right) d t \\
& \left.-\left\langle\left(\vec{x}^{(k)}\right)^{t}\left(\frac{\pi}{\sqrt{2}}\right)|O| \vec{x}^{(k)}\left(\frac{\pi}{\sqrt{2}}\right)\right\rangle-\int_{0}^{\frac{\pi}{\sqrt{2}}}\left(u_{x}^{(k)}\right)^{2}(t)+u_{y}^{(k)^{2}}(t)\right) d t \\
& =\left\langle\left(\vec{x}^{(k+1)}\right)^{t}\left(\frac{\pi}{\sqrt{2}}\right)-\left(\vec{x}^{(k)}\right)^{t}\left(\frac{\pi}{\sqrt{2}}\right)|O| \vec{x}^{(k+1)}\left(\frac{\pi}{\sqrt{2}}\right)-\vec{x}^{(k)}\left(\frac{\pi}{\sqrt{2}}\right)\right\rangle \\
& +2 \operatorname{Re}\left\langle\vec{x}^{(k+1)}\left(\frac{\pi}{\sqrt{2}}\right)-\vec{x}^{(k)}\left(\frac{\pi}{\sqrt{2}}\right)|O| \vec{x}^{(k)}\left(\frac{\pi}{\sqrt{2}}\right)\right\rangle \\
& +\int_{0}^{\frac{\pi}{\sqrt{2}}}\left(u^{(k+1)}{ }_{x}^{2}(t)+u^{(k+1)}{ }_{y}^{2}(t)\right) d t-\int_{0}^{\frac{\pi}{\sqrt{2}}}\left(u_{x}^{(k)^{2}}(t)+u_{y}^{(k)}(t)\right) d t . \\
& =\left\langle\left(\vec{x}^{(k+1)}\right)^{t}\left(\frac{\pi}{\sqrt{2}}\right)-\left(\vec{x}^{(k)}\right)^{t}\left(\frac{\pi}{\sqrt{2}}\right)|O| \vec{x}^{(k+1)}\left(\frac{\pi}{\sqrt{2}}\right)-\vec{x}^{(k)}\left(\frac{\pi}{\sqrt{2}}\right)\right\rangle \\
+\int_{0}^{\frac{\pi}{\sqrt{2}}}\left(\left(\frac{2}{\delta_{1}}\right.\right. & \left.-1)\left(u_{y}^{(k+1)}(t)-v^{(k)}(t)\right)^{2}+\left(\frac{2}{\eta_{1}}-1\right)\left(v^{(k)}(t)-u_{y}^{(k)}(t)\right)^{2}\right) d t \\
+\int_{0}^{\frac{\pi}{\sqrt{2}}}\left(\left(\frac{2}{\delta_{2}}\right.\right. & \left.-1)\left(u_{x}^{(k+1)}(t)-w^{(k)}(t)\right)^{2}+\left(\frac{2}{\eta_{2}}-1\right)\left(w^{(k)}(t)-u_{x}^{(k)}(t)\right)^{2}\right) d t \\
& >0
\end{aligned}
$$

\section{Conclusion}

In this paper we have devised a monotonic convergent algorithm, inspired in Rabitz et al, Krotov et al. and Maday-Turinici algorithms [3], to find the optimal controls to perform a unitary spin evolution of the state spin $1 / 2$ to state spin $-1 / 2$ in a minimal time and minimum cost for a two-level quantum system. The corresponding optimal controls and the minimum cost were calculated (Fig. 1 and Fig. 2, respectively) when there are two external electromagnetic fields. The results were compared with the analytical solution which was found using the Pontryagin Maximum Principle [5]. The proposed algorithm converges rapidly to known analytical solutions, which are sine and cosine [6]. The strategy yields good performances in the case study we have analyzed. Of course, a structured validation of the new algorithm is required and also is possible, in each step of the process, to splits the cost functions into fidelity and pulse energy. Finally, we consider important the implementation and the development of iterative numerical algorithms to solve quantum control problems in the case of quantum multi-levels.

\section{References}

[1] Romero-Meléndez, C., González-Santos, L. (2017). A Numerical Approximation Approach for Quantum Optimal Control of Two-Level Systems. International Journal of Applied Physics and Mathematics, 7(4). 
[2] Tannoudji, C., Diu, B., \& Laloe, F. (1978). Quantum Mechanics. V.1. Wiley-VCH.

[3] Maday, I., \& Turinici, G. (2003). New formulations of monotonically convergent quantum control algorithms. Journal of Chemical Physics, 118(18).

[4] D' Alessandro, D. (2007). Introduction to quantum control and dynamics. Ser. Applied Mathematics \& Nonlinear Science. Chapman \& Hall/CRC.

[5] Pontryagin, L., Boltyanskii, B., Gamkrelidze, R., \& Mishchenko, E. (1962). The Mathematical Theory of Optimal Processes. Wiley-Interscience, New York.

[6] D' Alessandro, D., \& Dahleh, M. (2001). Optimal control of two-level quantum systems. IEEE Transactions on Automatic Control, 46(6).

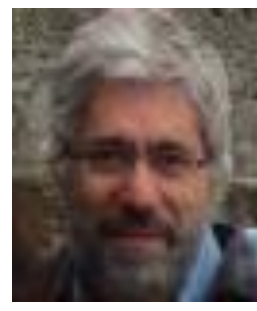

Cutberto Romero-Meléndez received the degree in mathematical physics from Institute National Polytechnic at Mexico, the master degree from the Metropolitan Autonomous University, at Mexico and the Ph.D. degree Trés Honorable in mathematics from the Bourgogne University at Dijon, France, in 1985, 1989 and 2004, respectively.

He is currently a full professor in the Department of Basic Sciences of the Metropolitan Autonomous University, at Mexico. His research interests include geometric control theory, optimal and nonlinear control theory, control of quantum mechanical systems, complexity in motion planning and path planning.

Dr. Romero belongs to the Mathematical Mexican Society, he is the Chairman of the Organizing Committee of the Multidisciplinary Congress of Sciences Applied in Latin America and he received an Academic Teaching Award in Engineering in 1994.

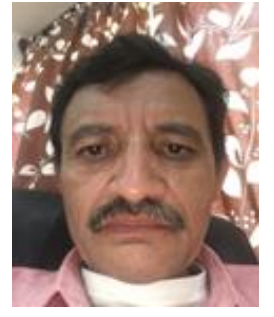

Leopoldo González-Santos received the degree of mathematical physics and the master's degree in computer science from the National Polytechnic Institute at Mexico in 1980 and 1999, respectively.

He is currently an academic technician in the Department of Functional Mapping of the Institute of Neurobiology of the National Autonomous University of Mexico as a developer of numerical methods for the analysis of structural and functional magnetic resonance imaging. His research interests include numerical methods theory, finite element method, data mining theory and neural networks. 